\title{
Developmental outcomes of children with Duarte galactosemia: exploring the bases of an apparent contradiction in the literature
}

\author{
Judith L. Fridovich-Keil, PhD ${ }^{1}{ }^{1}$, Grace Carlock, BA ${ }^{1}$, Claire D. Coles, PhD², Mary Ellen Lynch, PhD ${ }^{2}$, \\ Molly N. Millians, DEd ${ }^{2}$, Nancy L. Potter, PhD ${ }^{3}{ }^{3}$, Kimberly Powell, PhD, RDN (1) ${ }^{4}$, Peter Richards ${ }^{5}$, \\ Rani Singh, PhD, RD ${ }^{1}$ and Angela Wittenauer, MSN, $\mathrm{RN}^{1}$
}

Genetics in Medicine (2019) 21:2683-2685; https://doi.org/10.1038/s41436-019-0567-1

\begin{abstract}
INTRODUCTION
We submit this Comment to address an apparent contradiction in the literature concerning developmental outcomes of children with Duarte galactosemia (DG). Specifically, in 2009, Powell and colleagues published a report ${ }^{1}$ in this journal showing that children with DG were overrepresented in metropolitan Atlanta among 3- to 10-year-olds receiving speech-language intervention in public school. While indirect, this result raised the alarming possibility that children with DG might be at increased risk for difficulties with speech and/or language. In contrast, this year Carlock and colleagues published a report ${ }^{2}$ showing by direct assessment of speech-language outcomes in 350 children that no significant differences were observed between cases and controls. While different results are often reported from independent studies of human subjects, it can be informative to compare details of the studies to identify potential explanations for the apparent disparities.
\end{abstract}

\section{STUDY DESIGN AND CASE-CONTROL COHORTS}

The Powell study leveraged newborn screening, metabolic clinic, and other records to identify 75 infants with DG born between 1988 and 2001 in metropolitan Atlanta, of whom 59 were treated at Emory University and still living in metropolitan Atlanta 3-10 years later; these comprised the cases for the study. Infants not treated at Emory, or no longer living in metropolitan Atlanta at ages 3-10 years, were excluded. Controls for the Powell study were infants born in metropolitan Atlanta who survived to at least 1 year, were 3-10 years old in 1998, and were still living in metropolitan Atlanta.

Developmental outcomes and receipt of special services for cases were ascertained by linking the 59 names of children with DG in the study with each of two Centers for Disease Control and Prevention (CDC) databases covering the years 1996-2004 when the children would have been 3-10 years old: (1) the Metropolitan Atlanta Developmental Disabilities Surveillance Program, a population-based surveillance system for selected developmental disabilities; and (2) the Special Education Database of Metropolitan Atlanta (SEDMA), a database listing children in metropolitan Atlanta receiving special services in public schools. Prevalence of controls receiving special services was determined by dividing the number of 3- to 10-year-olds listed in SEDMA as receiving special services in $1998(13,187)$ by the total number of controls $(295,939)$.

Cases for the Carlock study were 206 children with DG, all 6-12 years old, recruited from 13 US states with the help of the newborn screening programs and metabolic clinics that diagnosed them as infants. Controls were 144 unaffected 6- to 12-year-olds from these same families. Speech-language outcomes for the Carlock study were determined by direct evaluation of each child by each of two experienced speech pathologists who were blinded to the case-control status of each child. Parents were also asked if their child had ever received speech-language intervention.

\section{STUDY RESULTS}

Although Powell and colleagues found that none of the 59 children with DG in their study had been diagnosed with intellectual disability, cerebral palsy, hearing loss, vision impairment, or an autism spectrum disorder, 5 of the 59 , or $8.5 \%$, had received special services for speech and/or language in school at some point when they were 3 to 10 years old. When the age range was narrowed to include only 8 -year-olds

\footnotetext{
${ }^{1}$ Department of Human Genetics, Emory University School of Medicine, Atlanta, GA, USA; ${ }^{2}$ Department of Psychiatry and Behavioral Sciences, Emory University School of Medicine, Atlanta, GA, USA; ${ }^{3}$ Department of Speech and Hearing Sciences, Washington State University, Spokane, WA, USA; ${ }^{4}$ Department of Human Sciences, North Carolina Central University, Durham, NC, USA; ${ }^{5}$ Center for the Study of Human Health, and Department of Psychology, Emory College, Atlanta, GA, USA. Correspondence:
} Judith L. Fridovich-Keil (jfridov@emory.edu) 
the prevalence of cases receiving speech-language intervention was recalculated as $5 / 33$, or $15.2 \%$.

For controls in the Powell study, the prevalence of metropolitan Atlanta 3- to 10-year-olds receiving special services for speech/language in 1998 was calculated as 13,187/ 295,939 or $4.5 \%$. Using the narrower age range, the prevalence of all metropolitan Atlanta 8-year-olds receiving speech-language intervention in public school in 1998 was $2264 / 38,328$ or $5.9 \%$. For both the larger and smaller age ranges considered, the prevalence of cases receiving speech-language intervention was greater than the prevalence of controls.

The Carlock study evaluated child participants using established instruments that yielded continuous or ordinal scores for 73 different outcomes representing five developmental domains. Fourteen of the 73 outcomes tested related to speech-language. No significant differences were seen between cases and controls, or among cases as a function of exposure to milk in infancy, for any of the outcomes tested. Consistent with this result, $10.3 \%$ of the 206 cases, and $11.2 \%$ of the 144 controls, reported having received speech-language intervention at some point in school. Further, the average duration of speech-language intervention was almost identical: 4.4 years for cases and 4.5 years for controls.

\section{STUDY STRENGTHS AND LIMITATIONS}

A major strength of the Powell study is that it was largely population based, albeit from one geographic area. Because subjects were not consented, there was no self-selection bias for participation.

However, cases and controls may have been to some extent mismatched. Specifically, because the D2 allele of GALT associated with DG is prevalent in populations of European ancestry, and almost absent from populations of African ancestry, ${ }^{3}$ few infants identified with DG are African American. For example, Georgia newborn screening data for the years 2006 to 2015 demonstrated that of 126 infants diagnosed with DG whose race was known (another 33 were race unknown and 10 were multiracial or other), $83 \%$ were white and only $9.5 \%$ were African American. In contrast, the percentage of African American newborns in the population screened was almost $34 \%$. This could be a contributing factor to results of the Powell study as prior reports document that receipt of speech-language intervention can be disparate by race $^{4,5}$ with African American children being underserved. ${ }^{6}$

A major strength of the Carlock study is the large number of both cases and controls directly tested by trained professionals. Another strength is that participating families came from 13 US states representing different regions of the country. Both cases and controls derived from the same set of families and were well matched for many factors including race and socioeconomic status.

A limitation of the Carlock study is that families were consented, so there may have been a self-selection bias, and participating families tended to be more highly educated than the general US population. ${ }^{7}$ It is possible that a study of children not subject to these biases might have yielded different results. However, any ascertainment biases in the Carlock study cohort should have been balanced between cases and controls.

\section{COMPARISON OF STUDY RESULTS WITH RELE- VANT DATA FROM OTHER SOURCES}

Finally, it is useful to compare the results of both the Powell and Carlock studies with relevant data from other sources. For example, according to the $\mathrm{CDC}^{4} 9.3 \%$ of US children ages 7 to 10 were reported in 2012 as having a communication disorder in the previous 12 months. Of communication disorders, speech-language difficulties were the most common. This means that close to $360 / 4000$ school children experienced a communication disorder, but the prevalence of DG is only about $1 / 4000$. Whether, in rare cases, DG causes a speech-language disorder, or in some cases a child with DG experiences an independent cause of speech-language difficulty, remains unclear.

It is also important to note that of children reported by the $\mathrm{CDC}$ as having a communication disorder, only about $2 / 3$ received intervention. ${ }^{4}$ That, on average, about one-third of US children said to have a problem did not receive intervention, and that this percentage differed across populations, ${ }^{4}$ highlights the challenge in defining child outcome based on receipt of special services in school. Specifically, whether or not a child receives services can be impacted by many factors, some related to the child and family, but many related to resources available in the school, the school system, and local or national regulations that may change over time.,

The CDC numbers listed above are similar to the $8.5 \%$ of 3to 10 -year-old cases, and higher than the $4.5 \%$ of 3 - to 10 year-old controls, reported from the Powell study as receiving speech-language intervention. Of note, an independent study also using the SEDMA database used by Powell reported an even lower percentage of children in metropolitan Atlanta receiving speech-language intervention in public schools $(3.88 \%)^{8}$

The CDC numbers listed above are also notably lower than the $10.3 \%$ of cases and $11.2 \%$ of controls reported as receiving speech-language intervention from the Carlock study. Of course, the geographies and time frames in which these numbers were derived differed, so that eligibility criteria and other factors may have also differed. Further, the Carlock study asked parents whether their child had ever received intervention for a speech-language problem; the CDC study asked parents only about intervention in the prior 12 months. Finally, as mentioned above, because the D2 allele of GALT underlying DG occurs predominantly in European populations, ${ }^{3}$ the Carlock study families, who were recruited on the basis of having at least one child with DG, included predominantly families of European ancestry.

\section{CONCLUSION}

Like all human subjects research, both the Powell and Carlock studies had strengths and limitations that may have 
contributed to their contrasting findings. Specific differences related to study design, potential ascertainment bias, matching versus mismatching case and control cohorts, and potential confounding of outcome variables. To answer the question of whether children with DG are at increased risk for developmental problems, all of these factors must be taken into account.

\section{ACKNOWLEDGEMENTS}

We are grateful to Stuart Shapira for his thoughtful comments and helpful discussion.

\section{DISCLOSURE}

The authors declare no conflicts of interest.

Publisher's note: Springer Nature remains neutral with regard to jurisdictional claims in published maps and institutional affiliations.

\section{REFERENCES}

1. Powell KK, Van Naarden Braun K, Singh RH, Shapira SK, Olney RS, Yeargin-Allsopp M. Long-term speech and language developmental issues among children with Duarte galactosemia. Genet Med. 2009;11:874-879.

2. Carlock G, Fischer ST, Lynch ME, et al. Developmental outcomes in Duarte galactosemia. Pediatrics. 2019;143:e20182516.

3. Carney $A E$, Sanders $R D$, Garza $K R$, et al. Origins, distribution and expression of the Duarte-2 (D2) allele of galactose-1-phosphate uridylyltransferase. Hum Mol Genet. 2009;18:1624-1632.

4. Black L, Vahratian A, Hoffman H. Communication disorders and use of intervention services among children age 3-17 years: United States, 2012. NCHS Data Brief. 2015;Jun(205):1-8.

5. Morgan PL, Farkas G, Hillemeier MM, Li H, Pun WH, Cook M. Crosscohort evidence of disparities in service receipt for speech or language impairments. Except Child. 2017;84:27-41.

6. Morgan P, Farkas G, Cook M, et al. Are black children disproportionately overrepresented in special education? A best-evidence synthesis. Except Child. 2017;83:181-198.

7. US Census Bureau. Quick facts. 2018. https://www.census.gov/ quickfacts/fact/table/US/PST045218.

8. Riehle-Colarusso T, Autry A, Razzaghi H, et al. Congenital heart defects and receipt of special education services. Pediatrics. 2015;136:496-504. 